\title{
Melanoma nasofaríngeo: caso clínico
}

\section{Nasopharyngeal melanoma: clinical case}

\author{
Miriam del Carmen Marrero R. ${ }^{1}$, María Sandra Domínguez S. ${ }^{1}$, María Soledad Cabrera $R .{ }^{1}$, \\ Sergio Miranda F. ${ }^{1}$, Marta González $V^{2}$
}

\section{Resumen}

El melanoma mucoso primario (MM) de la cavidad nasal y los senos paranasales sigue siendo un tumor maligno poco frecuente. El pico de incidencia se sitúa entre 50 y 60 años, siendo la epistaxis y la obstrucción nasal unilateral los síntomas más frecuentes. La resección quirúrgica es el tratamiento primario con radioterapia y quimioterapia adyuvante si es necesario, el pronóstico depende de la extensión. Presentamos el caso de una paciente mujer de 81 años con hemoptisis y halitosis de un año de evolución. La nasofibrolaringoscopia mostró una lesión parduzca, excrecente, irregular y friable, en el borde derecho del rodete tubárico derecho. El resultado histopatológico fue MM nasofaríngeo. Perfil inmunohistoquímico: S100 +, MelanA +, HMB45 +, SOX10 +, AE1AE3 negativo. El PET-CT mostró una hipercaptación a nivel del rodete tubárico derecho, sin evidencia de adenopatías o metástasis a distancia. Se realizó la resección tumoral completa mediante abordaje endoscópico. El Comité de Oncología del Melanoma decidió que no era necesario un tratamiento adyuvante debido a la resección completa de la lesión y considerando la edad y el deterioro cognitivo de la paciente.

Palabras clave: melanoma de la mucosa, cirugía endoscópica, rodete tubárico, nasofibroscopía, epistaxis.

\begin{abstract}
Primary mucous melanoma (MM) of the nasal cavity and paranasal sinuses remains a rare malignancy. The peak of incidence is between 50 and 60 years old, being epistaxis and unilateral nasal obstruction the most frequent symptoms. Surgical resection is the primary treatment with radiotherapy and adjuvant chemotherapy if necessary, the prognosis depends on the spreading. We present the case of an 81-year-old female patient attending for hemoptysis and halitosis of a year of evolution. The nasofibrolaryngoscopy showed a brownish, excrescent, irregular and friable lesion, outgrowth of the right tube rim. Histopathology result was nasopharyngeal MM. Immunohistochemical profile: $\mathrm{S} 100+\mathrm{Me}-$ lanA +, HMB45 +, SOX10 +, AE1AE3 negative. PET-CT showed a hypercaptation at the level of the right tube rim, without evidence of adenopathies or distant metastases. Complete tumor resection was performed by endoscopic surgery. Melanoma Oncology Committee decided no need of adjuvant therapy due to the complete resection of the lesion and considering the age and cognitive deterioration of the patient.
\end{abstract}

Keywords: Mucosal melanoma, endoscopic surgery, tube rim, nasofibroscopy, epistaxis.
'Servicio de

Otorrinolaringología, Hospital Universitario de Gran Canaria, Dr. Negrín. Las Palmas de Gran Canaria, España. ${ }^{2}$ Servicio de Anatomía Patológica, Hospital Universitario de Gran Canaria, Dr. Negrín. Las Palmas de Gran Canaria. España.

Los autores declaran no tener conflictos de interés. Recibido el 18 de mayo de 2020. Aceptado el 1 de diciembre de 2020.

Correspondencia: Miriam del Carmen Marrero R. Calle Heredad de Agua $n^{\circ} 5$ Las Palmas de Gran Canaria. España.

Email: miriammarrero93@ gmail.com

\section{Introducción}

El melanoma nasofaríngeo es un tipo extremadamente raro y agresivo de melanoma mucoso de cabeza y cuello (MMCC) $)^{1,2}$. Los melanomas de las mucosas de la cavidad nasal y el tracto nasosinusal representan el $4 \%$ de los melanomas mucosos de cabeza y cuello, y el $4 \%$ de las neoplasias del tracto nasal/nasofaríngeo ${ }^{3}$. $\mathrm{Su}$ incidencia es ligeramente superior a partir de los 60 años ${ }^{1,2}$. Los síntomas más frecuentes de presentación como epistaxis, hipoacusia y congestión nasal se desarrollan lentamente; por lo tanto, muchos pacientes reciben un diagnóstico tardío, lo que reduce la supervivencia ${ }^{1,4,5}$. Debido a la rareza del melanoma mucoso, la 
mayoría de los estudios sobre esta enfermedad son informes de una sola institución con un número limitado de pacientes, y los datos sobre los resultados y la epidemiología son escasos ${ }^{6}$. El diagnóstico se basa en hallazgos anatomopatológicos e inmunohistoquímicos ${ }^{7}$.

El tratamiento de elección es la resección quirúrgica, mientras que la radioterapia y la quimioterapia sirven para controlar la enfermedad local y metastásica ${ }^{1}$. A pesar de los avances en las capacidades de diagnóstico y los enfoques de tratamiento, el pronóstico sigue siendo malo y la tasa de supervivencia a los 5 años es del 10\%-30\%, en parte debido a las altas tasas de recurrencias y metástasis a distancia ${ }^{7}$.

\section{Caso Clínico}

Presentamos el caso de una mujer de 81 años con antecedentes personales de glaucoma, hipertensión arterial, deterioro cognitivo y meningioma petroclivial no quirúrgico, en seguimiento por el servicio de neurología; acude a consultas externas de otorrinolaringología por hemoptisis y halitosis de un año de evolución. En el examen físico por nasofibrolaringoscopia, se observa una lesión parduzca, excrecente, irregular y friable, con restos hemáticos alrededor, inmediatamente anterior al rodete tubárico en la fosa nasal derecha (Figura 1A).

Se realizó una biopsia, con resultado histopatológico positivo para melanoma mucoso nasofaríngeo (MM); con un perfil inmunohistoquímico: $\mathrm{S} 100+$, Mela$\mathrm{nA}+, \mathrm{HMB} 45+$, SOX10 +, AE1AE3 negativo (Figuras $1 \mathrm{~B}$ y $2 \mathrm{~A}$ ). El informe de tomografía computada (TC) reveló la presencia de una lesión de 2,0 x 1,3 cm en el lado derecho del cavum, sospechosa de malignidad, y el PETTC confirmó una hipercaptación en la pared derecha de la nasofaringe, a nivel del rodete tubárico, compatible con malignidad, en relación con el diagnóstico histopatológico de melanoma, sin evidencia de adenopatías o metástasis a distancia (Figura 2B).

Se realiza resección quirúrgica mediante abordaje endoscópico incluido rodete tubárico, con márgenes libres de $0,5 \mathrm{~cm}$. No se procede a la colocación de drenaje transtimpánico en el acto quirúrgico a la espera de informe histopatológico de márgenes de resección y en el posterior seguimiento en consultas externas no ha sido necesario ante la ausencia de disfunción tubárica ipsilateral. El Comité de Oncología del Melanoma determina que no es necesaria una terapia adyuvante debido a la resección completa de la lesión, con márgenes libres, sin infiltración de la mucosa adyacente (S100 negativa) y la edad y el deterioro cogniti-

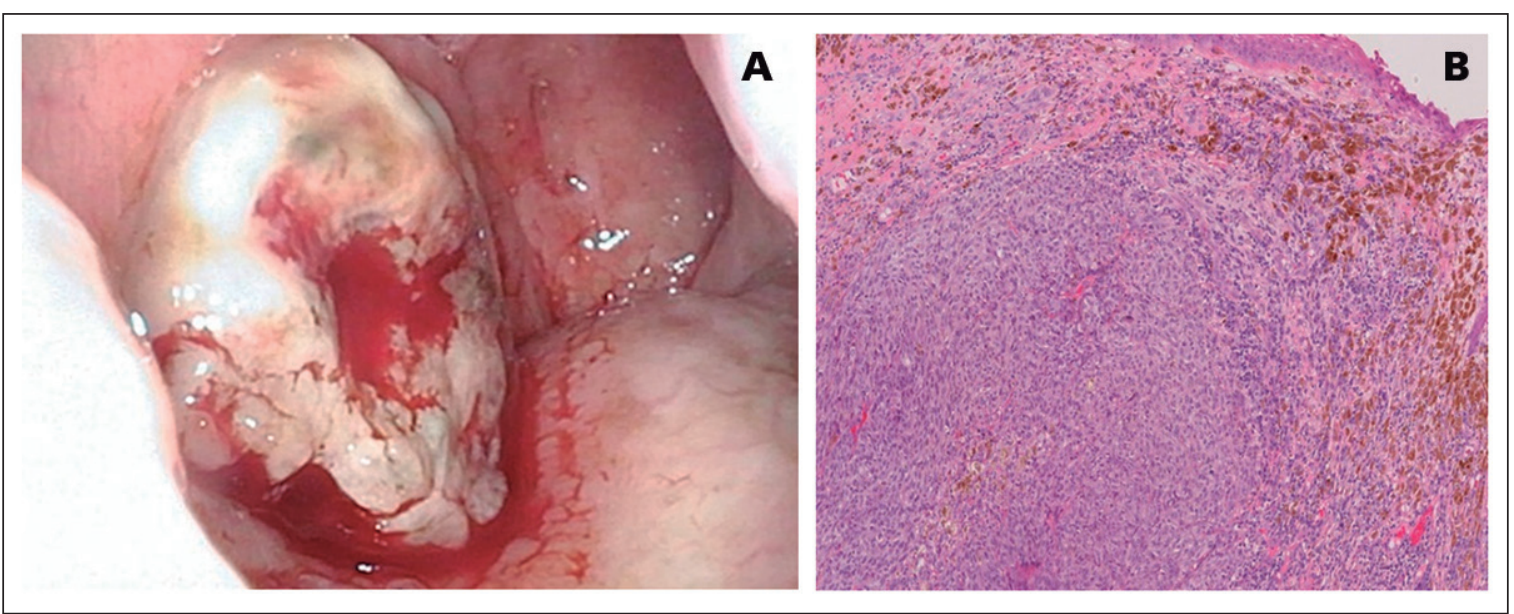

Figura 1. A) A la izquierda, melanoma nasofaríngeo. Imagen mediante visualización fibroscópica: lesión pardusca, excrecente, irregular y friable, con restos de sangre a su alrededor, inmediatamente antes del rodete tubárico derecho. B) A la derecha, la tinción de hematoxilina y eosina, muestra células tumorales con pigmentos de melanina y un epitelio superficial (fotomicrografía digital, aumento $\times 10$ ). 


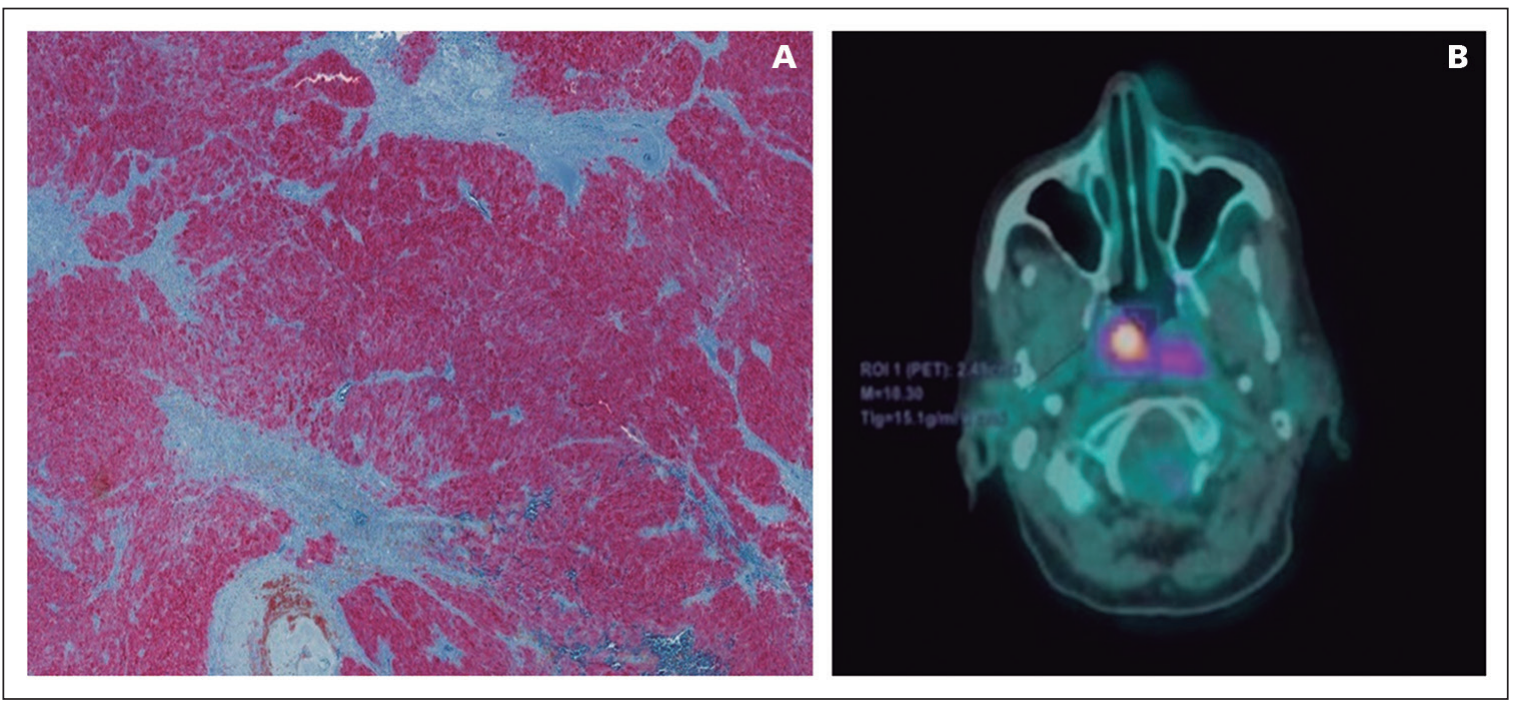

Figura 2. A) A la izquierda, las células tumorales son difusamente positivas (tinción roja) para HMB-45, un marcador común para melanoma (fotomicrografía digital, aumento $\times$ 4). B) A la derecha, imagen PET-TC: hipercaptación en la pared derecha de la nasofaringe, a nivel del rodete tubárico, compatible con malignidad, en relación con la lesión denominada melanoma.

vo de la paciente. Un año después de la cirugía, nuestra paciente continúa siendo revisada periódicamente en nuestra unidad, sin incidentes ni evidencia de recurrencia locorregional en el momento actual.

\section{Discusión}

Las neoplasias malignas del tracto nasosinusal representan aproximadamente el 3\% de los tumores malignos de cabeza y cuello ${ }^{8}$. El MM primario de la cavidad nasal y los senos paranasales sigue siendo una neoplasia maligna poco frecuente y poco estudiada, lo que constituye el 0,5\%-2\% de los melanomas aerodigestivos ${ }^{9}$. La cavidad nasal es la ubicación anatómica más frecuente con respecto a la cabeza y el cuello, específicamente, el tabique nasal ${ }^{2,10}$. El pico de incidencia se sitúa entre los 50 y 60 años $^{1,2}$, siendo la epistaxis ${ }^{6,11}$ y la obstrucción nasal unilateral ${ }^{4}$ los síntomas más frecuentes ${ }^{10}$. Aunque nuestro paciente acudió al Servicio de Otorrinolaringología por primera vez por esputo hemoptoico, presentó episodios de epistaxis unilateral antes de la cirugía. La evaluación de la extensión de la lesión es esencial y, en ocasiones, se utilizan técnicas como la PET-CT para descartar me- tástasis a distancia ${ }^{12}$. La resección quirúrgica es la modalidad de tratamiento primario para el MM y la radioterapia adyuvante después de la cirugía ofrece un mejor control locorregional incluso para tumores pequeños ${ }^{13}$, aunque su pronóstico depende de la propagación, y la supervivencia general es pobre independientemente del enfoque del tratamiento ${ }^{10}$. No están relacionados con la exposición a la radiación ultravioleta, a diferencia de los melanomas cutáneos $^{14-16}$.

En cuanto a la técnica quirúrgica, en muchos estudios defienden la rinotomía paralateronasal como la técnica quirúrgica de elección. Para Díaz Molina y cols. ${ }^{14}$, en un estudio retrospectivo de 17 pacientes, la técnica quirúrgica de elección fue la rinotomía paralateronasal (10 de los 12 pacientes operados) en lugar de la cirugía endoscópica nasosinusal. Sin embargo, el artículo de Yin y cols. ${ }^{16}$, un estudio retrospectivo de 54 pacientes divididos en dos grupos (27 pacientes tratados con cirugía endoscópica y otros 27 pacientes tratados con cirugía abierta), describe que estadísticamente no hubo diferencias significativas en la recurrencia local, supervivencia libre de enfermedad o supervivencia general. Los resultados de la cirugía endoscópica nasosinusal son superiores en el tiempo quirúrgico, menores complicaciones 
posquirúrgicas y mínimas cicatrices en la cara del paciente $y$, por lo tanto, mejora la calidad de vida posoperatoria.

La quimioterapia y la inmunoterapia se reservan como tratamiento paliativo, siendo la inmunoterapia una gran promesa en el tratamiento futuro de esta patología ${ }^{18}$, con el empleo de bacilo de Calmette-Guérin (BCG) directo sobre el tumor como método inmunosupresor más utilizado. Posteriormente, el interferón ha supuesto otra alternativa para el tratamiento de MM y, actualmente, fármacos como el vemurafenib (inhibidor del $\mathrm{BRAF}^{\mathrm{V}}{ }^{\mathrm{C} 00 \mathrm{E}}$ ) o el ipilimumab (anticuerpo monoclonal que bloquea la acción CTLA-4) se están destapando como terapias novedosas y prometedoras ${ }^{14}$.

Peng y cols. ${ }^{1}$, en una serie de 44 casos que diferenciaron los melanomas de la cavidad nasal (37), los senos paranasales (5) y la nasofaringe (2), concluyeron que sólo el estadio clínico afectaba el pronóstico, mientras que la ubicación no se correlacionó con la supervivencia a los 5 años $^{18}$. Lo mismo se observa en el estudio de Bijan y cols. ${ }^{19}$, un estudio retrospectivo de 19 pacientes, con una supervivencia a 5 años del 23\%, donde la etapa clínica inicial del MM es el único valor pronóstico estadísticamente significativo. Un análisis del registro del Programa de Vigilancia, Epidemiología y Resultados Finales (SEER) entre 1973 y 2007 identificó varios predictores independientes de una supervivencia general deficiente y libre de enfermedad. Dichos factores incluyen la localización en nasofaringe/seno paranasal, edad mayor de 70 años y metástasis a distancia, estando los dos primeros presentes en nuestra paciente $^{19}$. Cabe destacar que hasta el $50 \%$ de los pacientes con melanoma mucoso de cabeza y cuello pueden desarrollar metástasis a distancia en cerebro, hígado y pulmones ${ }^{20} \mathrm{y}$, a pesar del tratamiento, el pronóstico del MM sigue siendo muy pobre, con una alta tasa de recurrencia local y metástasis a distancia, así como una tasa de supervivencia a 5 años del 10\%-30\% según los estudios, por lo que es necesaria una estrecha vigilancia de estos pacientes ${ }^{15,21-23}$.

\section{Conclusión}

El MM ubicado en cavidad nasal o nasofaringe es una entidad poco común y difícil de diagnosticar debido a sus síntomas clínicos inespecíficos. Un examen nasofibrolaringoscópico es esencial para el diagnóstico, siendo la inmunohistoquímica y las pruebas de imagen imprescindibles para evaluar la extensión de la lesión. El tratamiento debe planificarse a través de un comité de oncología para discutir diferentes opciones terapéuticas, siendo en la actualidad, la cirugía el tratamiento de elección.

\section{Bibliografía}

1. Alves IS, Berriel LG, Alves RT, et al. Sinonasal Melanoma: A Case Report and Literature Review. Case Rep Oncol Med. 2017;2017:8201301. doi: 10.1155/2017/8201301.

2. Núñez VC, Rojas KC, Serdio JA. Melanoma maligno mucoso de cavidad nasal. Rev Otorrinolaringol Cir Cabeza Cuello. 2015; 75:152-156.

3. Thompson LD, Wieneke JA, Miettinen M. Sinonasal tract and nasopharyngeal melanomas: a clinicopathologic study of 115 cases with a proposed staging system. Am J Surg Pathol. 2003; 27(5):594611.

4. McLean N, Tighiouart M, Muller S. Primary mucosal melanoma of the head and neck. Comparison of clinical presentation and histopathologic features of oral and sinonasal melanoma. Oral Oncol. 2008; 44(11):1039-1046.

5. Dauer EH, Lewis JE, Rohlinger AL. Sinonasal melanoma: a clinicopathologic review of 61 cases. Otolaryngol Head Neck Surg. 2008; 138(3):347-352.

6. Marcus DM, Marcus RP, Prabhu RS, et al. Rising incidence of mucosal melanoma of the head and neck in the United States. J Skin Cancer. 2012; 2012:231693. doi: 10.1155/2012/231693.

7. Manolidis S, Donald PJ. Malignant mucosal melanoma of the head and neck: review of the literature and report of 14 patients. Cancer. 1997; 80(8):1373-1386

8. López F, Grau JJ, Medina JA. Consenso español para el tratamiento de los tumores nasosinusales. Acta Otorrinolaringol Esp. 2017; 68(4):226-234.

9. Poissonnet G, Castillo L, Dassonville O, Ettore F. Malignant nasosinus melanomas. Literature review on 12 cases. Rev Laryngol Otol Rhinol (Bord). 1997; 118(3):155-61.

10. Gilain L, Houette A, Montalban A. Melanoma of the mucosa of the nasal cavity and sinuses. Eur Ann Otorhinolaryngol Head Neck Dis. 2014; 131(6):365-69.

11. Djiguimdé W, Diomandé I, Bonnin N. Malignant melanoma of the nasal septum, a rare tumor, 
occurring in a 54-year-old patient after hereditary retinoblastoma treatment. Int Med Case Rep J. 2016; 9:25-28.

12. Maza-Solano JM, Sánchez-Gómez S, Herrero-Salado TM. Nasopharyngeal melanoma: an uncommon entity. Acta Otorrinolaringol Esp. 2014; 65 (5):314-16.

13. Christopherson K, Malyapa RS, Werning JW, Morris CG, Kirwan J, Mendenhall WM. Radiation therapy for mucosal melanoma of the head and neck. Am J Clin Oncol. 2015;38(1):87-89. doi: 10.1097/ COC.0b013e31828d73bf.

14. Díaz-Molina JP, Rodrigo-Tapia JP, Llorente-Pendas JL. Rhinosinus mucous melanomas. Review of 17 cases. Acta Otorrinolaringol Esp. 2008; 59(10):489-93.

15. Ziolkowska M, Bien S, Klimas A. The epidemiology and clinical features of malignant melanoma located in the nasal cavity and sinuses. Six case analysis. Otolaryngol Pol. 2008; 62(4):500-3.

16. Yin G, Guo W, Chen X. Prognosis of endoscopic surgery and traditional open resection in the melanoma of the mucosa of the nasal cavity and paranasal sinus. Melanoma Res. 2019; 29(1):47-52.

17. Sayaka K, Mamoru T, Nasahiro T. Mucosal melanoma of the head and neck. Experimental and Therapeutic Medicine. 2011; 2: 907-910.

18. Peng RQ, Wu GH, Chen WK. Clinical characteristics and prognosis of the primary melanoma of the nasal mucosa. Report of 44 cases. Ai Zheng. 2006; 25(10):1284-86.

19. Jethanamest D, Vila PM, Sikora AG, Morris LG. Predictors of survival in mucosal melanoma of the head and neck. Ann Surg Oncol. 2011; 18(10)27482756.

20. Meleti M, Leemans CR, Vescovi P. Head and neck mucosal melanoma: experience with 42 patients, with emphasis on the role of postoperative radiotherapy. Head Neck. 2008; 30 (12):1543-1551.

21. McLean N, Tighiourat M, Muller S. Primary mucosal melanoma of the head and neck. Comparison of clinical presentation and histopathologic features of oral and sinonasal melanoma. Oral Oncol. 2008; 44:1039-46.

22. Saïd C, Joseph G, David M. Unknown primary nasopharyngeal melanoma presenting as severe recurrent epistaxis and hearing loss following treatment and remission of metastatic disease: A case report and literature review. Int J Surg Case Rep. 2015; 10: 232-235.

23. Manola J, Atkins M, Ibrahim J. Prognostic factors in metastatic melanoma: a pooled analysis of Eastern Cooperative Oncology Group trials. J Clin Oncol. 2000; 18(22):3782-3793. 